BGU-PH-97/06

\title{
Graceful Exit and Energy Conditions in String Cosmology
}

\author{
Ram Brustein and Richard Madden \\ Department of Physics, Ben-Gurion University, Beer-Sheva 84105, Israel
}

\begin{abstract}
String cosmology solutions are examined in a generalized phase-space including sources representing arbitrary corrections to lowest order string-dilatongravity effective action. We find a set of necessary conditions for a graceful exit transition from a dilaton-driven inflationary phase to a radiation dominated era. We show that sources allowing such a transition have to violate energy conditions similar to those appearing in singularity theorems of general relativity. Since familiar classical sources, excepting spatial curvature, obey these energy conditions we conclude that a generic graceful exit in string cosmology requires a new effective phase of matter. Our results clarify and generalize previous analyses and enable us to critically reexamine proposed non-singular cosmologies.
\end{abstract}




\section{Introduction}

Duality symmetries of string cosmology equations [1, 2, 3], suggest a mechanism [1, 4] for inflationary evolution. This mechanism is based on the fact that cosmological solutions to string dilaton-gravity come in duality-related pairs, the plus branch $(+)$, and the minus branch $(-)$ [5]. The $(+)$ branch, even in the absence of potential energy, has inflationary solutions in which the Hubble parameter increases with time. The minus branch $(-)$ can be connected smoothly to a standard Friedman-Robertson-Walker (FRW) decelerated expansion of the universe with constant dilaton. The idea (so called "pre-big-bang" scenario) is that evolution of the universe starts from a state of very small curvature and coupling and then undergoes a long phase of dilaton-driven kinetic inflation described by the $(+)$ branch and at some later time joins smoothly standard radiation dominated cosmological evolution, thus giving rise to a singularity free inflationary cosmology.

A key issue in the realization of this idea has been the nature of the graceful exit transition from the initial phase of dilaton-driven kinetic inflation to the subsequent standard radiation dominated evolution, in particular, revealing conditions under which such a transition is allowed or proving that under certain conditions a graceful exit transition is forbidden. In [5] it was argued, and later proved [6], that such a transition cannot occur while curvature was below the string scale and the string coupling was still weak, leading to the conclusion that an intermediate "string phase" of high curvature (previously suggested as a possibility [1, 4]) or strong coupling is actually required [7]. Recently, this was a subject of intense activity, some investigations reinforcing and expanding the domain of validity of the original conclusions [8], some resorting to non-perturbative quantum effects to assist a transition [9], with varying degrees of success, while some have claimed that additional fields with certain form of interactions are required [10, 11, 12].

Our approach is to use an effective description in terms of sources that represent arbitrary corrections to the lowest order equations, depriving us the ability to obtain concrete solutions, but allowing analysis of classes of solutions and properties of effective sources. A solution of the "true equations" necessarily satisfies the effective equations, even if the corrections to the lowest order equations are large, as long as the whole framework does not break down. There are different conformal frames in which one can describe the equations. These are related by local field redefinitions which, suppos- 
edly, do not affect physical observables [13]. We perform the analysis in the string frame $(\underline{S} \mid)$ and in the "lowest order Einstein frame" $(\underline{E})$. We are able to relate necessary conditions for graceful exit to energy conditions appearing in singularity theorems of Eintein's general relativity [14], and show that a successful exit requires violations of one of the weakest of the energy conditions, the null energy condition (NEC).

Classical sources, such as perfect fluids with "reasonable" equation of state, minimally coupled fields, etc, tend to obey NEC and therefore we conclude that a new kind of effective source is required to allow generic graceful exit. We discuss various sources and comment about their relevance and we also put in context recent proposals offering non-singular evolution. We confirm our analysis by identifying the NEC violating sources as resulting from special interaction terms between more or less conventional sources. None of the proposals seem to fit the full set of requirements for a good string cosmology as defined in the text.

\section{Effective string cosmology}

String theory effective action in 4 dimensions takes the following form

$$
S_{\text {eff }}^{S \mid}=\int d^{4} x\left\{\sqrt{-g}\left[\frac{e^{-\phi}}{16 \pi \alpha^{\prime}}\left(R+\partial_{\mu} \phi \partial^{\mu} \phi\right)\right]+\frac{1}{2} \mathcal{L}_{m}\left(\phi, g_{\mu \nu}, \ldots\right)\right\},
$$

where $g_{\mu \nu}$ is the 4 -d metric and $\phi$ is the dilaton, the effective action is written here in the string frame $(\underline{S\rfloor})$. The "matter" Lagrangian $\mathcal{L}_{m}$ may contain corrections to the lowest order 4-d action coming from a variety of sources, such as $\alpha^{\prime}$ higher derivative corrections, string quantum corrections, additional fields, denoted by ..., extra dimensions, string matter, etc.

We are interested in solutions to the equations of motion derived from the action (2.1) of the FRW type with vanishing spatial curvature (nonvanishing spatial curvature may be included as an effective source in the equations) $d s^{2}=-d t_{S}^{2}+a_{S}^{2}(t) d x_{i} d x^{i}$ and $\phi=\phi(t)$. To allow solutions of this

type the "matter" energy-momentum tensor $T_{\mu \nu}=\frac{1}{\sqrt{-g}} \frac{\delta \mathcal{L}_{m}}{\delta g^{\mu \nu}}$, should have the form $T_{\nu}^{\mu}=\operatorname{diag}(\rho,-p,-p,-p)$.

Of the three equations of motion and one conservation equation only three are independent and we choose the following as the independent set

$$
3 H_{S}^{2}+\frac{1}{2} \dot{\phi}^{2}-3 H_{S} \dot{\phi}=\frac{1}{2} e^{\phi} \rho_{S}
$$




$$
\begin{aligned}
\dot{H}_{S}+3 H_{S}^{2}-H_{S} \dot{\phi} & =\frac{1}{2} e^{\phi}\left(p_{S}+\Delta_{\phi} \mathcal{L}_{m}\right) \\
\dot{\rho}_{S}+3 H_{S}\left(\rho_{S}+p_{S}\right) & =-\Delta_{\phi} \mathcal{L}_{m} \dot{\phi},
\end{aligned}
$$

where $\Delta_{\phi} \mathcal{L}_{m}=\frac{1}{2} \frac{1}{\sqrt{-g}} \frac{\delta \mathcal{L}_{m}}{\delta \phi}, H_{S}=\dot{a}_{S} / a_{S}$, and we have fixed our units such that $16 \pi \alpha^{\prime}=1$. The constraint equation (2.2) may be used to rewrite equations (2.2-2.4) in the form

$$
\begin{aligned}
\dot{\phi} & =3 H_{S} \pm \sqrt{3 H_{S}^{2}+e^{\phi} \rho_{S}} \\
\dot{H}_{S} & = \pm H_{S} \sqrt{3 H_{S}^{2}+e^{\phi} \rho_{S}}+\frac{1}{2} e^{\phi}\left(p_{S}+\Delta_{\phi} \mathcal{L}_{m}\right) \\
\dot{\rho}_{S}+3 H_{S}\left(\rho_{S}+p_{S}\right) & =-\Delta_{\phi} \mathcal{L}_{m} \dot{\phi} .
\end{aligned}
$$

Our designation of the branches $(+)$ and $(-)$ corresponds to the sign choice in these equations. Note that the constraint requires that $e=3 H_{S}^{2}+e^{\phi} \rho_{S} \geq 0$ ( $e<0$ is a classically forbidden region) and that a change of branches can only occur where $e=0$. It is this surface in phase space that we refer to as the "egg" [5, 6]. Also notice that in the absence of strong sources the $\dot{H}$ equation shows that the $(+)$ branch solutions are unstable $(\operatorname{sign}(H)=$ $\operatorname{sign}(\dot{H})$ ), evolving to future singularities and conversely the $(-)$ branch will evolve from past singularities.

We may perform a field redefinition to the "lowest order Einstein frame" $(\underline{E} \mid) g_{\mu \nu} \rightarrow G_{\mu \nu}=e^{-\phi} g_{\mu \nu}$ and $\phi \rightarrow \varphi=\phi$ which transforms the lowest order dilaton-gravity action into Einstein's gravity and a minimally coupled scalar field, canonically normalized, $S_{e f f}^{\frac{E}{e f}}=\int d^{4} x\left\{\sqrt{-G} \frac{R}{16 \pi G_{N}}+\frac{1}{2} \widetilde{\mathcal{L}}_{m}\left(\varphi, G_{\mu \nu}, \ldots\right)\right\}$, where $\widetilde{\mathcal{L}}_{m}\left(\varphi, G_{\mu \nu}\right)=\mathcal{L}_{m}\left(\varphi, G_{\mu \nu} e^{\varphi}\right)-\sqrt{-G} \partial_{\mu} \varphi \partial^{\mu} \varphi$, so that we have included the dilaton kinetic energy in the definition of the matter Lagrangian in $\underline{E}$ but not in $\underline{S\rfloor}$. Looking for solutions of the FRW type $d s^{2}=-d t_{E}^{2}+a_{E}^{2}(t) d x_{i} d x^{i}$ and $\varphi=\varphi(t)$, the equations of motion reduce to the standard set, $H_{E}^{2}=$ $\frac{1}{6} \rho_{E}, \ldots$, etc. (we have chosen units in which $16 \pi G_{N}=1$ ). A particularly interesting linear combination of the $t t$ and $i i$ equations which we will use later is the following

$$
\dot{H}_{E}=-\frac{1}{4}\left(\rho_{E}+p_{E}\right)
$$

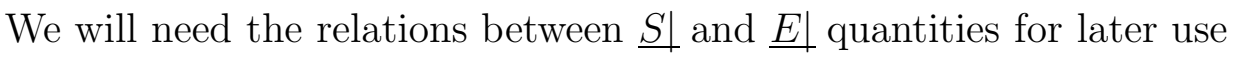

$$
\begin{aligned}
d t_{E} & =e^{-\phi / 2} d t_{S} ; a_{E}=e^{-\phi / 2} a_{S} ; H_{E}=e^{\phi / 2}\left(H_{S}-\frac{1}{2} \dot{\phi}\right) \\
p_{E} & =e^{2 \phi} p_{S}+\frac{1}{2} e^{\phi} \dot{\phi}^{2} ; \rho_{E}=e^{2 \phi} \rho_{S}+\frac{1}{2} e^{\phi} \dot{\phi}^{2} .
\end{aligned}
$$


To construct a physically interesting cosmological evolution we require certain conditions on the asymptotic behaviour of solutions. In our setup we have to specify boundary conditions for four of the five functions $\left\{H_{S}, \phi, \dot{\phi}, \rho_{S}, p_{S}\right\}$ and determine the boundary values of the remaining function subject to the constraint (2.2) and branch choice. The boundary conditions should guarantee that the problems of the standard model of cosmology are solved as in all models of inflation and that later evolution does agree with the observed universe (see also [15]). The initial conditions for $t \rightarrow-\infty H_{S}>0, \phi \ll 0, \dot{\phi}>$ $0, \rho_{S} \simeq p_{S} \simeq 0$ and the choice of an initial $(+)$ branch guarantee that the solution will emerge as an unstable perturbation of the vacuum, which can then evolve into a long period of dilaton dominated kinetic inflation without reaching the very strong coupling regime $\phi \gg 0$. The final phase we require to be ordinary radiation dominated FRW cosmology with a constant dilaton of intermediate value, $H>0, \phi \simeq 0, \dot{\phi}=0, \rho=3 p$. Note that in this case $\underline{S\rfloor}$ and $\underline{E\rfloor}$ quantities are proportional to each other and we therefore drop the subscript $S$. The final state of radiation, for which $T_{\mu}^{\mu}=\rho-3 p=0$, is consistent with a constant decoupled dilaton, which in turn implies that we are on a $(-)$ branch. Thus at least one branch change event from $(+)$ to $(-)$ has to occur in the course of the evolution.

In addition, we require that throughout the evolution, even during the string phase, all components of curvature invariants, $\phi, \rho, p$, and their derivatives remain finite. We do not allow a singular field redefinition for which new curvature invariants derivatives and sources are finite. The reason for this requirement is to guarantee that there are some physical observables which survive the evolution. This requirement is similar in spirit to the bounded curvature idea in [16], and less similar to ideas about smoothing and resolution of singularities suggested in string theory context [17].

Finally we note that our definition of the "lowest order Einstein frame" $(\underline{E})$ coincides with the true Einstein frame with decoupled dilaton and gravity near our initial and final conditions. In some of the models we examine,

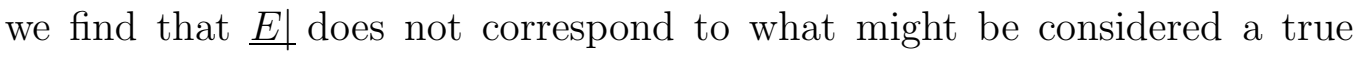
Einstein frame, since dilaton-gravity couplings have been absorbed into the effective $\rho$ and $p$. 


\section{Branch change and exit}

At least one branch change event from $(+)$ to $(-)$ has to occur in the course of the evolution, as explained in the previous section. We focus our attention on the last branch change which has to be from $(+)$ to $(-)$. Recall the egg variable $e=3 H_{S}^{2}+\rho_{S} e^{\phi}$. A necessary condition for a branch change to occur is that the evolution of the solution touch the egg, the surface in phase space defined by $e=0$, which requires that $\rho_{S}<0$ in some region of parameter space. Continuity of the solution then forces a branch change. The region of negative $\rho_{S}$ is divided into two parts, $H_{S}>0$ (above the egg) and $H_{S}<0$ (below the egg) separated by the classically forbidden region $e<0$. Notice that above the egg $\dot{\phi}>0$ and below the egg $\dot{\phi}<0$.

Using the equations of motion we can show that

$$
\dot{\sqrt{e}}= \pm\left[3 H_{S}^{2}+\frac{1}{2} \rho_{S} e^{\phi}-\frac{1}{2} \Delta_{\phi} \mathcal{L}_{m} e^{\phi}\right]
$$

For an immediate application of eq.(3.1) consider a $(+)$ solution approaching the egg. For it to hit the egg $e$ must decrease to zero, so $\sqrt{e}<0$ just before the egg is hit. If the sources are not coupled to the dilaton, then $\Delta_{\phi} \mathcal{L}_{m}=0$ and we can write $\sqrt{e}=3 H_{S}^{2}+\frac{1}{2} \rho_{S} e^{\phi}=e-\frac{1}{2} e^{\phi} \rho_{S}$. But this quantity is positive just before an egg hit, since we have observed $\rho_{S}<0$ there. In this case the egg repels $(+)$ branch solutions and we conclude branch change is not possible. The matter sources have to be rather strongly coupled to the dilaton for a branch change to be possible.

We proceed to derive a further "global" necessary condition for exit based on the requirement that the last branch change will really be the last branch change. Recall that if the evolution of the solution touches the egg, a branch change must occur. We therefore require that the evolution of the solution does not touch the egg anymore after the last branch change. It is enough to make sure that no further branch changes occur until the "moment of escape", after which $\rho_{S}$ is always positive and an additional branch change is impossible. We discuss the generic situation, deferring discussion of possible pathological cases as in [6] to another opportunity. To derive the global condition, following [6], we use eq.(2.3) to eliminate $\Delta_{\phi} \mathcal{L}_{m}$ from eq.(3.1)

$$
\dot{\sqrt{e}}= \pm\left[-\dot{H}_{S}+H_{S} \dot{\phi}+\frac{1}{2} e^{\phi}\left(\rho_{S}+p_{S}\right)\right] .
$$


Assuming a successful escape, we integrate eq.(3.2) from the moment of branch change $t_{\text {hit }}$, at which $e=0$ and $\dot{\phi}=3 H_{S}$ to the moment of escape $t_{\text {escape }}$, at which $\rho_{S}=0, e=\sqrt{3} H_{S}$ and $\dot{\phi}=(3-\sqrt{3}) H_{S}$

$$
\int_{t_{\text {hit }}}^{t_{\text {escape }}} d t\left[-\sqrt{e}+\dot{H}_{S}\right]=\int_{\phi\left(t_{\text {hit }}\right)}^{\phi\left(t_{\text {escape }}\right)} H_{S} d \phi+\int_{t_{\text {hit }}}^{t_{\text {escape }}} d t \frac{1}{2} e^{\phi}\left(\rho_{S}+p_{S}\right) .
$$

Performing the integration on the left-hand-side we obtain

$$
-\left[(\sqrt{3}-1) H_{S}\left(t_{\text {escape }}\right)+H_{S}\left(t_{\text {hit }}\right)+\mathcal{A}\right]=\int_{t_{\text {hit }}}^{t_{\text {escape }}} d t \frac{1}{2} e^{\phi}\left(\rho_{S}+p_{S}\right),
$$

where $\mathcal{A}=\int_{\phi\left(t_{\text {hit }}\right)}^{\phi\left(t_{\text {escape }}\right)} H_{S} d \phi$ is the positive area of the domain bounded by the solution and $\phi$-axis shown in figure 1. Note that the left-hand-side of eq.(3.4) is negative. In fact, it probably has a rather large magnitude because $H_{S}$ is likely to be rather large throughout this epoch. We conclude that a necessary condition for a successful escape is that

$$
\int_{t_{\text {hit }}}^{t_{\text {escape }}} d t e^{\phi}\left(\rho_{S}+p_{S}\right)<0 .
$$

For some specific cases this condition was derived in [6].

A global condition in $\underline{E}$ is obtained as follows. We may show, using relations (2.7) and the condition $e=0$, that the value of $H_{E}$ at the moment of "egg-hit" is negative

$$
\begin{aligned}
H_{E}\left(t_{\mathrm{hit}}\right) & =e^{\phi\left(t_{\mathrm{hit}}\right) / 2}\left(H_{S}\left(t_{\mathrm{hit}}\right)-\frac{1}{2} \dot{\phi}\left(t_{\mathrm{hit}}\right)\right) \\
& =e^{\phi\left(t_{\mathrm{hit}}\right) / 2}\left(H_{S}\left(t_{\mathrm{hit}}\right)-\frac{3}{2} H_{S}\left(t_{\mathrm{hit}}\right)\right) \\
& =-\frac{1}{2} e^{\phi\left(t_{\mathrm{hit}}\right) / 2} H_{S}\left(t_{\mathrm{hit}}\right)<0 .
\end{aligned}
$$

We may also show, using relations (2.7) and the condition $e=\sqrt{3} H_{S}$, that the value of $H_{E}$ at the moment of escape is positive

$$
H_{E}\left(t_{\text {escape }}\right)=e^{\phi\left(t_{\text {escape }}\right) / 2}\left(H_{S}\left(t_{\text {escape }}\right)-\frac{1}{2} \dot{\phi}\left(t_{\text {escape }}\right)\right)
$$



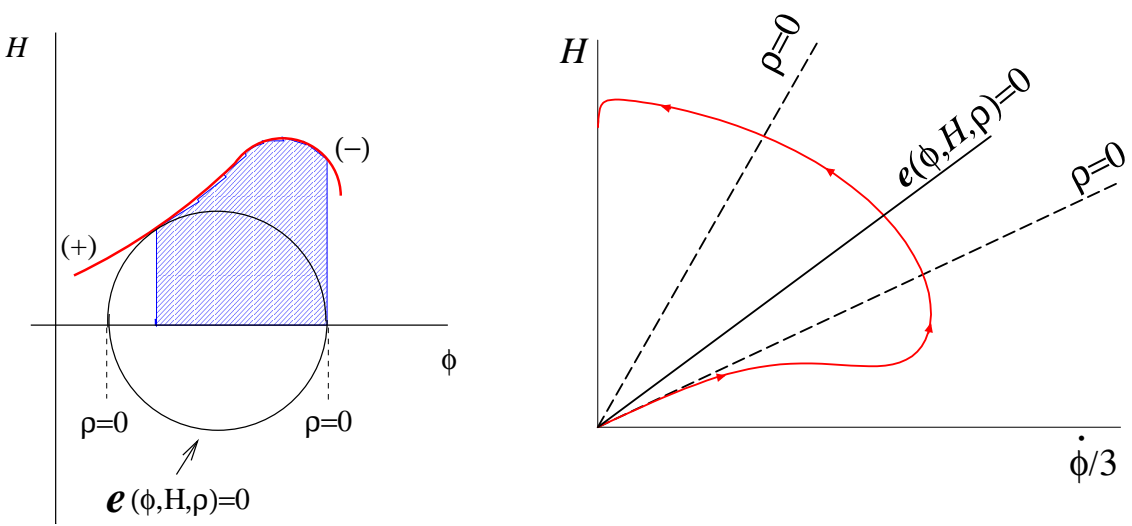

Figure 1: Shown are two (hypothetical) examples of a complete graceful exit in $\underline{S}$. The one on the left is shown in $(\phi, H)$ plane with an arbitrary origin for the $\phi$ axis. A transition from $(+)$ branch to $(-)$ branch occurs when the solution hits the curve $e(\phi, H, \rho)=0$ (the egg) and is then followed by a successful escape to a point where $\rho_{S}=0$. The shaded area $\mathcal{A}=\int H d \phi$ is always positive since $\phi$ is increasing and $H>0$. The one on the right is shown in the $(\dot{\phi}, H)$ plane with the axes origins at $(0,0)$. The solution begins asymptotically near the lower dashed line which is $\rho=0$ (vacuum) for the $(+)$ branch. The solution then crosses this line developing $\rho<0$ and makes a branch change crossing the solid line $e=0$. Finally it completes a graceful exit to the $\rho>0$ region by crossing the upper dashed line which is $\rho=0$ for the $(-)$ branch and reaches $\dot{\phi}=0$ and radiation domination.

$$
\begin{aligned}
& =e^{\phi\left(t_{\text {escape }}\right) / 2}\left(H_{S}\left(t_{\text {escape }}\right)-\frac{3-\sqrt{3}}{2} H_{S}\left(t_{\text {escape }}\right)\right) \\
& =e^{\phi\left(t_{\text {escape }}\right) / 2} \frac{\sqrt{3}-1}{2} H_{S}\left(t_{\text {escape }}\right)>0 .
\end{aligned}
$$

Therefore the total change in $H_{E}$ is positive $\Delta H_{E}=\int_{t_{\mathrm{hit}}}^{t_{\text {escape }}} d t_{E} \dot{H}_{E}>0$, which implies, using eq.(2.6), that $-\frac{1}{4} \int_{t_{\text {hit }}}^{t_{\text {escape }}} d t_{E}\left(\rho_{E}+p_{E}\right)>0$. We conclude that a necessary condition for a successful escape is that

$$
\int_{t_{\text {hit }}}^{t_{\text {escape }}} d t_{E}\left(\rho_{E}+p_{E}\right)<0 .
$$


Soon we will relate energy conditions (3.5), (3.8) to those used in singularity theorems of general relativity and discuss their significance, but for now we would like to discuss the relationship of a bounce in $\underline{E}$ to branches in $\underline{S}$.

A bounce in $\underline{E}$ is a transition from a contracting to an expanding universe $H_{E}<0 \Rightarrow H_{E}>0$. We wish to refine the relationship between a bounce in $\underline{E l}$ and a branch change and exit. Recall that $H_{E}=e^{\phi / 2}\left(H_{S}-\frac{1}{2} \dot{\phi}\right)$ and that for $(+)$ branch $\dot{\phi}-3 H_{S}>0$ and for $(-)$ branch $\dot{\phi}-3 H_{S}<0$. Considering solutions for which $H_{S}>0$ always, we obtain the following classification

$$
\begin{aligned}
& H_{E}<0 \Rightarrow \begin{cases}\dot{\phi}>3 H_{S} & \Rightarrow(+) \text { branch } \\
2 H_{S}<\dot{\phi}<3 H_{S} & \Rightarrow(-) \text { branch. }\end{cases} \\
& H_{E}>0 \Rightarrow(-) \text { branch. }
\end{aligned}
$$

Conversely, (always keeping $H_{S}>0$ )

$$
\begin{aligned}
& (+) \text { branch } \Rightarrow H_{E}<0 \\
& (-) \text { branch } \Rightarrow \begin{cases}\dot{\phi}<2 H_{S} & \Rightarrow H_{E}>0 \\
2 H_{S}<\dot{\phi}<3 H_{S} & \Rightarrow H_{E}<0 .\end{cases}
\end{aligned}
$$

The conclusion is that a bounce does not necessarily imply a branch change because a bounce can occur while the solution remains a (-) branch solution, and that a branch change does not necessarily imply a bounce because we have seen that $H_{E}$ is negative in the region around the branch change point. The bounce is only guaranteed if the solution proceeds to exit.

Let us summarize now the set of necessary conditions for implementing successful exit

- Initial conditions of a $(+)$ branch and $H_{S}, \dot{\phi}>0$ require $H_{E}<0$.

- A branch change from $(+)$ to $(-)$ has to occur while $H_{E}<0$. At that point $e=3 H_{S}^{2}+e^{\phi} \rho_{S}$ has to vanish and therefore $\dot{e}$ just before the branch change has to be negative, and positive just after.

- A successful escape and exit completion requires a bounce in $\underline{E}$ after the branch change has occurred, ending up with $H_{E}>0$.

- Further evolution is required to bring about a radiation dominated era in which the dilaton effectively decouples from the "matter" sources. 
We repeat for emphasis that a bounce in $\underline{E}$ is not a sufficient condition for an exit, and as already shown in [5, 6] a branch change is not a sufficient condition for an exit. We also state the trivial fact that necessary conditions are not sufficient conditions and therefore even if they are satisfied they do not ensure successful exit transition.

\section{$4 \quad$ Energy conditions}

We wish to relate the energy conditions in eqs.(3.5, 3.8) to those that appear in singularity theorems of general relativity 14. The energy conditions that are most relevant to the present discussion are the local null energy condition (NEC) and its global version, the averaged null energy condition (ANEC) [18], a subject of some recent investigations [19]. The NEC requires that $T_{\mu \nu} k^{\mu} k^{\nu} \geq 0$ for all null vectors $k^{\mu}$ and the ANEC requires that $\int T_{\mu \nu} k^{\mu} k^{\nu} d \lambda \geq 0$, where $\gamma$ is a null curve, $k^{\mu}$ is a tangent (null) vector and $\lambda$ is a generalized affine parameter. For $T_{\nu}^{\mu}=\operatorname{diag}(\rho,-p,-p,-p)$ the NEC reduces to $\rho+p \geq 0$ and the ANEC reduces to $\int_{\gamma}(\rho+p) d \lambda \geq 0$. The NEC is one of the weakest energy conditions and its violation implies a violation of the weak, dominant, strong, and generic energy conditions.

In $\underline{E l}$, condition (3.8) states that a necessary condition for an exit transition is that the NEC is violated for some range of time. In $\underline{S\rfloor}$ condition (3.5) similarly implies that $\rho_{S}+p_{S}<0$ for some range of time. In $\underline{S} \rho+p$ can be negative even if in $\underline{E}$ both $\rho$ and $p$ are positive $\rho_{E}+p_{E}=e^{2 \phi}\left(\rho_{S}+p_{S}\right)+e^{\phi} \dot{\phi}_{S}^{2}$, therefore condition (3.8) seems to be a more restrictive condition.

Our conclusion is that the NEC has to be violated to allow a graceful exit transition, implying that an effective source which enables graceful exit must invalidate the conditions under which the classical singularity theorems of general relativity were proved, as expected since we now have a non-singular cosmology. Energy conditions are arguably the weakest link in the chain of arguments leading to singularity theorems, in particular, we are not aware of any general argument that forbids from first principles NEC violating sources. In fact, violations of NEC are expected to occur due to quantum effects, as in the celebrated example of Hawking radiation of black holes, although their strength in some specific cases is under debate [19], and spatial curvature is a classical effective source that violates NEC (see below). Energy conditions 


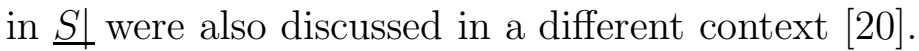

\section{Sources}

We now turn to discuss possible sources and their role in graceful exit transition, paying some attention to suggestions in the literature.

First, let us point out that our results generalize and reinforce the conclusions of [5, 6, 8] that found that adding fields and sources "off the shelf" (including all types of stringy matter) will not facilitate graceful exit since they obey NEC. Also, attempts to tinker with minimally coupled fields to produce a negative $\rho+p$ tend to create ghosts or tachyons, unacceptable if classical stability and quantum unitarity are required. Obviously, other kinds of effective sources are necessary.

Effective sources that naturally appear in string theory are

- Higher derivatives classical $\left(\alpha^{\prime}\right)$ corrections. Because of technical problems related to on-shell conditions, string theory is not very specific about the coefficients of higher derivative terms, and in particular, at the moment, there is no stringy principle able to constraint the effective $\rho+p$ of these terms. As we discuss below, in some cases they do provide negative $\rho+p$.

- Quantum corrections which manifest themselves as higher derivative corrections with a specific dilaton dependence, or in modification of the form of the coupling of the dilaton and gravity to other fields. It may well be possible to devise ad-hoc modifications that will allow an exit transition. However, it turns out that large classes of models, including most of those that appeared in the literature have some property that prevents graceful exit from happening. In a different context, It has been argued [19 that quantum NEC violations are generic, however, their strength in various cases is under debate. Note that Hawking radiation of black holes allowing the area of event horizons to shrink is a prime example of such a quantum effect.

A more radical departure from our framework consists of allowing Euclidean solutions describing classically forbidden tunneling transitions. We have nothing to say about this possibility, clearly outside the scope of our current investigations. 
- Extra dimensions. As already discussed in 50 the effective contribution of simple contracting or expanding extra dimensions is positive to both $\rho$ and $p$, making them a graceful exit suppressing source.

- Spatial curvature is perhaps the most obvious source with negative $\rho+p$. Recall that in $\underline{E} \rho_{\text {curv }}=-6 k / a^{2}(t)$ and $p_{\text {curv }}=2 k / a^{2}(t)$ where $k=0, \pm 1$, and therefore $\rho_{\text {curv }}+p_{\text {curv }}=-4 k / a^{2}(t)$. Thus, for $k=+1$, $\rho_{\text {curv }}+p_{\text {curv }}<0$. Combining spatial curvature with another off the shelf source, a cosmological constant yields cosmologies of the general form $a(t)=\cosh (t)$ with the negative $\rho+p$ from the curvature doing the job of reversing the contracting deSitter solution to the expanding one. We do have some reservations about using spatial curvature in this context since one of the jobs that a dilaton-driven inflationary phase does best is to erase initial spatial curvature. So if the universe successfully inflates we don't expect curvature to be a strong source when its help is required for graceful exit.

In [7], following the conclusions of [5, 6] the necessity of an intermediate string phase was recognized. The simplest possible extrapolation of the solution was suggested, $H_{S}=$ const, $\dot{\phi}=$ const. The simple extrapolation was taken up more seriously in [12], showing that indeed there is an all order solution of this type and furthermore that this "simple string phase" can be reached continuously from the $(+)$ branch initial conditions. Our results show that it is impossible to complete a graceful exit transition during a simple string phase. Recall from relations (2.7) that $H_{E}=e^{\phi / 2}\left(H_{S}-\frac{1}{2} \dot{\phi}\right)$, and therefore if $H_{S}$ and $\dot{\phi}$ are constants, the sign of $H_{E}$ cannot change during this phase. An examination of the numerical results of [12] (for $\mathrm{d}=3$ ) reveals that a branch change induced by some higher derivative terms has indeed occurred, but that $H_{E}$ is negative throughout and that a simple string phase solution represents a singular collapse in a finite time in $\underline{E}$. As the authors point out, with the still growing dilaton, quantum corrections may aid the transition to a completed graceful exit.

Other analyses [10, 11], presenting non-singular cosmologies do not use the desired boundary conditions which degrades somewhat their relevance. In [10 $H_{E}>0$ throughout the whole evolution, and therefore their evolution cannot exhibit a branch change, indeed, their solution is always a (-) branch solution. In 111 the initial conditions are such that $H_{S}<0, \dot{\phi}<0$ 
in the $(+)$ branch and "final conditions" $H_{S}>0, \dot{\phi}<0$ in the $(-)$ branch. However, the solution in 11] does exhibit branch changes and bounces, the Gauss-Bonnet with its specific coupling to the extra modulus field supplying the effective NEC violating source. Further examination also shows the dilaton has a very little influence in driving the system. Asymptotically these solutions are dominated by terms coming from the spatial curvature and the coupling between the modulus and Gauss-Bonnet terms so initial conditions are nowhere near the dilaton-gravity perturbative vacuum, making these solutions irrelevant to the envisioned graceful exit transition.

Finally, we look at examples of non-singular cosmologies without specifically stringy motivations [21]. These can be constructed from singular Einstein frame evolutions by choice of an appropriate conformal scaling, which dictates the non-minimal coupling for the scalar. Again, this coupling produces NEC violating sources in $\underline{S}$ and $\underline{E}$ but, of course, not in the true Einstein frame. So, while appearing non-singular, they cannot solve the problems addressed by inflation such as the horizon problem [22].

\section{Conclusion}

A singularity free cosmology requires the violations of the classic singularity theorems of general relativity. We have found that a graceful exit transition from a dilaton-driven inflationary phase to a radiation dominated era that allows a non-singular string cosmology must be induced by an effective source that violates one of the weakest energy conditions used in these theorems, the null energy condition (NEC). We examine various proposed non-singular cosmologies and find NEC violating sources induced by different forms of interactions, but conclude that none of these are directly relevant to graceful exit in string cosmology.

We are unaware of any fundamental physics principle that forbids or requires the existence of NEC violating sources. Although most known classical sources do obey NEC and therefore cannot induce an exit, spatial curvature is effectively an NEC violating source and there is evidence that quantum mechanical violations of NEC are generic and related to trace anomalies, a common quantum effect. A prime example of a quantum phenomenon that violates NEC is Hawking radiation of black holes that allows the shrinking

of the area of event horizons, even though this is forbidden by singularity 
theorems. With this in mind, we feel that the need for violations of NEC to accomplish the graceful exit should not be taken as evidence that it is a forbidden transition, but rather that it may involve different physics than expected.

\section{Acknowledgment}

This research is supported in part by the Israel Science Foundation administered by the Israel Academy of Sciences and Humanities. We thank Howard Burton and Nemanja Kaloper for discussions about [21].

\section{References}

[1] G. Veneziano, Phys. Lett. B265 (1991) 287.

[2] K.A. Meissner and G. Veneziano, Phys. Lett. B267 (1991) 33;

K.A. Meissner and G. Veneziano, Mod. Phys. Lett. A6 (1991) 3397;

A. Sen, Phys. Lett. B271 (1991) 295;

M. Gasperini and G. Veneziano, Phys. Lett. B277 (1992) 256;

K.A. Meissner, hep-th/9610131.

[3] A.A. Tseytlin and C. Vafa, Nucl. Phys. B372 (1992) 443.

[4] M. Gasperini and G. Veneziano, Astropart. Phys. 1 (1993) 317.

[5] R. Brustein and G. Veneziano, Phys. Lett. B329 (1994) 429.

[6] N. Kaloper, R. Madden and K.A. Olive, Nucl. Phys. B452 (1995) 667.

[7] R. Brustein, M. Gasperini, M. Giovannini and G. Veneziano, Phys. Lett. B361 (1995) 45.

[8] E.J. Copeland, A. Lahiri and D. Wands, Phys. Rev. D50 (1994) 4868;

J. Levin, Phys. Rev. D51 (1995) 462;

J. Levin, Phys. Rev. D51 (1995) 1536;

E.J. Copeland, A. Lahiri and D. Wands, Phys. Rev. D51 (1995) 1569;

J. Levin, Phys. Lett. B343 (1995) 69;

N. Kaloper, R. Madden and K.A. Olive, Phys. Lett. B371 (1996) 34; 
R. Easther, K. Maeda and D. Wands, Phys. Rev. D53 (1996) 4247;

J.E. Lidsey, gr-qc/9605017;

N. Kaloper, hep-th/9609087;

J.E. Lidsey, gr-qc/9609063;

R. Poppe and S. Schwager, hep-th/9610166;

A. Lukas, Burt A. Ovrut and D. Waldram, hep-th/9610238.

[9] M. Gasperini, J. Maharana and G. Veneziano, Nucl. Phys. B472 (1996) 349;

A. Lukas and R. Poppe gr-qc/9603047;

S.-J. Rey, Phys. Rev. Lett. 77 (1996) 1929;

M. Gasperini and G. Veneziano, Phys. Lett. B387 (1996) 715;

M.P. Dabrowski and C. Kiefer, hep-th/9701035;

J. Maharana, S. Mukherji and S. Panda, hep-th/9701115;

A. Buonanno, M. Gasperini, M. Maggiore and C. Ungarelli, hepth/9701146.

[10] I. Antoniadis, J. Rizos and K. Tamvakis, Nucl. Phys. B415 (1993) 497.

[11] R. Easther and K. Maeda, Phys. Rev. D54 (1996) 7252.

[12] M. Gasperini, M. Maggiore and G. Veneziano, hep-th/9611039.

[13] M. Gasperini and G. Veneziano, Mod. Phys. Lett. A8 (1993) 3701.

[14] S.W. Hawking and G.F.R. Ellis, The large scale structure of space-time, Cambridge University Press, Cambridge, England, 1973;

S.W. Hawking, hep-th/9409195.

[15] G. Veneziano, hep-th/9512091.

[16] V. Mukhanov and R. Brandenberger, Phys. Rev. Lett. 68 (1992) 1969.

[17] E. Kiritsis and C. Kounnas, Phys. Lett. B331 (1994) 51;

A.A. Tseytlin, Phys. Lett. B334 (1994) 315;

E. Martinec, Class. Quant. Grav. 12 (1995) 941;

A. Lawrence and E. Martinec, Class. Quant. Grav. 13 (1996) 63;

F. Larsen and F. Wilczek, hep-th/9610252.

[18] T.A. Roman, Phys. Rev. D37 (1988) 546. 
[19] M. Visser, Phys. Lett. B349 (1995) 443;

E. Flanagan and R. Wald, Phys. Rev. D54 (1996) 6233;

F. Antonsen and K. Bormann, hep-th/9702009.

[20] R. Brustein, unpublished;

S. Kar, hep-th/9611122.

[21] S.K. Rama, hep-th/9611223;

S.K. Rama, hep-th/9701154.

[22] Y. Hu, E.J. Weinberg and M.S. Turner, Phys. Rev. D49 (1994) 3830;

J. Levin and K. Freese, Nucl. Phys. B421 (1994) 635. 